\title{
BMJ open PROSpER: PReferences for the Organisation of acute health Services for oldER people: protocol for a mixed methods study
}

\author{
Kirsten Howard, ${ }^{1}$ Glenn Arendts, ${ }^{1,2,3}$ Stephen Jan, ${ }^{4}$ Matthew Beck ${ }^{5}$
}

To cite: Howard K, Arendts G, Jan S, et al. PROSpER: PReferences for the Organisation of acute health Services for oldER people: protocol for a mixed methods study. BMJ Open 2012;2: e001081. doi:10.1136/ bmjopen-2012-001081

- Prepublication history for this paper is available online. To view these files please visit the journal online (http:// dx.doi.org/10.1136/ bmjopen-2012-001081).

Accepted 28 February 2012

This final article is available for use under the terms of the Creative Commons Attribution Non-Commercial 2.0 Licence; see http://bmjopen.bmj.com

${ }^{1}$ School of Public Health, University of Sydney, Sydney, New South Wales, Australia

${ }^{2}$ Centre for Clinical Research in Emergency Medicine, Western Australian Institute for Medical Research, Perth, Western Australia, Australia ${ }^{3}$ School of Primary, Aboriginal and Rural Health Care, University of Western Australia, Crawley, Western Australia, Australia

${ }^{4}$ The George Institute for Global Health, Camperdown, New South Wales, Australia ${ }^{5}$ Institute for Transport and Logistics Studies, University of Sydney, Sydney, New South Wales, Australia

Correspondence to Dr Kirsten Howard; kirsten. howard@sydney.edu.au

\section{ABSTRACT}

Background: Organisation of acute care services for people living in residential aged care facilities (RACF) is a complex area of health policy. For people living in RACF, the emergency department is often used to provide acute care; needs of RACF residents, however, are not always well met. Alternative models of delivering care must be acceptable to a variety of stakeholders; however, little is known about the values and preferences that people attach to aspects of how and where care is delivered.

Methods/design: The PROSpER Study examines people's preferences for the organisation of acute healthcare services for older people in RACF. The authors aim to (1) determine which factors influence preferences of residents, carers and providers for how and where acute care is delivered and (2) determine the relative importance of these factors and the acceptable trade-offs between them. Qualitative and quantitative methods will be used. One-on-one interviews will be conducted with RACF residents, their families, staff of RACF and emergency department staff. A discrete choice study will then be designed to quantitatively assess preferences for alternative models of care delivery. Approximately 600 respondents from three respondent groups will be surveyed: older people living in RACF, family members of aged care residents and staff of RACF. A mixed logit model will be used; results will be expressed as parameter estimates ( $\beta$ ) and odds of choosing one option over an alternative. Trade-offs between attributes will also be calculated.

Ethics and dissemination: The PROSpER Study has been approved by the University of Sydney, Human Research Ethics Committee (Protocol numbers 10653 and 14382) and Royal Perth Hospital Ethics Committee (reference 2009/045). Results will be published in peer-reviewed scientific journals and via conference presentations; a newsletter will also be provided to study participants. A stakeholder roundtable will also be held to discuss the results.

\section{BACKGROUND}

As of June 2008, there were around 157000 people living permanently in residential aged care facilities (RACF) in Australia, a 19\%

\section{ARTICLE SUMMARY}

Article focus

- To assess the preferences of older people living in residential aged care facilities (RACF), their families and staff in relation to the provision of acute health care services for older Australians in RACF.

- To determine what factors most influence the preferences of these stakeholders for how and where acute care is delivered.

- To determine the relative importance of these factors and the trade-offs between them.

\section{Key messages}

- The organisation of acute healthcare services for older people living in RACF is a complex area of health policy involving considerations of clinical, economic, ethical and legal issues. For older people living in RACF, the emergency department is frequently used to provide acute care services. While emergency department generally works well if people have a short-term problem that can be resolved with a one-off intervention, the needs of people from RACF, often with chronic disease, multiple complex health problems and frailty are less well met.

- Alternative models of delivering acute care need to be acceptable to the residents, family and RACF staff; however, little is known about the values and preferences that people attach to aspects of how and where care is delivered or the trade-offs between various aspects of care that people are willing to make.

- This study will use best practice qualitative and quantitative methods for preference elicitation (a discrete choice experiment) to assess the preferences and acceptable trade-offs of RACF residents, their families and staff for alternative healthcare delivery models.

increase over the prior decade. Almost all $(96 \%)$ were aged 65 years or over and approximately $75 \%$ required high-level care. ${ }^{1}$ People living in RACF often represent the frailest and most vulnerable subgroup of older Australians and as a consequence have considerable healthcare needs. 


\section{ARTICLE SUMMARY}

Strengths and limitations of this study

- The strengths of the study are that it is the first study to use discrete choice methods to examine preferences for alternative models of acute healthcare service delivery in multiple stakeholder groups: residents of RACF, their families and RACF staff.

- The limitation is that it is conducted in one country, Australia, and thus its generalisability may be limited by the prevailing model of acute healthcare service delivery.

The organisation of acute healthcare services for older people living in RACF is a complex area of health policy involving major clinical, economic, ethical and legal issues. $^{2}{ }^{3}$ Acute healthcare is defined as the necessary treatment of significant illness or injury for a short period of time ${ }^{4}$ and is usually rendered in an ambulatory setting (eg, general practice), hospital emergency department (ED) or other short stay facility.

\section{The ED model of care}

For older people living in RACF, the ED is frequently used to provide acute care services, with up to 1.5 transfers to ED per RACF bed per year. ${ }^{5}{ }^{6}$ Acute clinical care in the ED is generally of high clinical standard, and the ED offers some other advantages for patients: it is a 'one-stop-shop' with $24 \mathrm{~h}$ access to diagnostic services such as pathology and radiology and easy access to specialty consultation services, it is generally a no cost option and patients (and their relatives) may feel reassured by being treated in a hospital environment. However, it is now widely asserted that this practice is financially unsustainable, given shifts in population demographics. It has been estimated that up to half of all transfers to ED from RACF are avoidable, ${ }^{7}$ and $20 \%$ of older people in public hospitals would be more appropriately cared for outside an acute hospital setting. ${ }^{5}$ In ED the additional care needs of people with chronic disease, multiple complex health problems and frailty are relatively poorly met. People living in RACF typically fall into all these categories, and ED-based care is associated with a number of negative consequences.

After an ED visit, older people are at increased risk of medical complications, functional decline and poorer quality of life. $^{8}{ }^{9}$ The ED environment is often overcrowded, noisy and lacks privacy and is unsuited to the needs of vulnerable older patients. ${ }^{10}$ The physical layout of the ED, designed for rapid assessment, stabilisation and management, focuses on open space, no walls and the need for sharing of space during busy periods. The priorities of ED care and the physical design are often not aligned with special needs of older people. ${ }^{11}$ ED staff have limited time to assist with the additional care needs (such as help with toileting and spending long periods of time answering questions) that may be required by older people. ${ }^{11} 12$ Complications frequently arise during patient transfers from RACF to the ED. ${ }^{13}$ For example, the common practice of using bladder catheters can increase the risk of delirium and infection, ${ }^{14}{ }^{15}$ and older patients are at substantially increased risk of falling because of multiple gait hazards such as linoleum floors, unnatural lighting and equipment. ${ }^{11}$ Some limited data suggest that residents and carers may prefer acute care be delivered in the RACF setting, with a known environment and staff and without the need for ambulance transfer to and from the ED ${ }^{16} \mathrm{ED}$ care, at least for some illnesses, is also expensive and resource intensive compared with comparable care delivered in a primary care setting. ${ }^{17}$

\section{Alternatives to the ED model}

Alternatives to ED-based care for people living in RACF have been proposed. Most commonly these involve 'outreach' programmes where care is provided in RACF instead of transferring residents to the ED. ${ }^{18}$ Various practice models include enhanced primary care through general practitioner or nurse practitioner-led care, ${ }^{19} 20$ supplementation of staffing within the RACF with highly trained multidisciplinary geriatric teams,${ }^{21}$ the use of remote telephone or telemedicine advice lines for RACF staff $^{22}$ and 'hospital in the nursing home' programmes ${ }^{23}$ or specialist geriatric EDs. ${ }^{11} 24$

Non-ED-based services will avoid many of the negative consequences of ED discussed above but may have potential disadvantages: nursing staff in RACF have high existing patient to staff ratios, some staff may not have the skills or resources to help manage acutely ill people, and alternative models may not offer the $24 \mathrm{~h}$ access that is necessary in some situations. Though many arguments are made in favour of reducing hospitalisation rates in older people in RACF, the success of policies to achieve this will rely on the support of patients, carers and providers. Alternative models of delivering acute care need to be designed in such a way as to be effective, cost-effective and ultimately acceptable. Until we understand the values and preferences people attach to aspects of how and where care is delivered, any new policy to reorganise acute healthcare services and reduce reliance on ED care will not be responsive to community expectations and is likely to fail.

\section{Aims}

We will assess the preferences of older people living in RACF, their families and staff in relation to the provision of acute healthcare services for older Australians in RACF. Specifically we will

- determine what factors most influence the choices of these stakeholders for how and where acute care is delivered.

- determine the relative importance of these factors and the trade-offs between them.

Through a better understanding of the value that various stakeholders attach to the different components of healthcare provision, we can optimise design of acute care strategies for RACF patients such that provision of care is both efficient and responsive to patient and family preferences. 


\section{METHODS/DESIGN}

\section{Overview of approach and methods}

This study will explore preferences and views on the delivery and organisation of acute care services for older Australians. A mixed methods approach will be used. One-on-one interviews will be conducted, and discrete choice experiments (DCEs) will be used to quantitatively assess preferences for alternative care delivery options. The discrete choice study will follow the ISPOR Guidelines for Good Research Practices for conjoint analysis in health. $^{25}$

\section{Discrete choice experiments}

DCEs involve surveys in which respondents are asked to choose between alternative policies or treatments defined by a set of differing attributes. This method is becoming more widely used in health as a means of quantifying patient and consumer preferences for healthcare policies and programmes. ${ }^{25-29}$ The method is based on the idea that goods and services, including healthcare services, can be described in terms of a number of separate attributes or factors. The levels of attributes are varied systematically in a series of questions and respondents choose the option that they prefer for each question. People are required to choose the option that is most preferred or has the highest 'value'. From these choices, a mathematical function is estimated which describes numerically the value that respondents attach to different choice options. Other data collected in the survey, including attitudinal questions and sociodemographic information, may also enter the value functions as explanatory variables. Ultimately, DCE studies can determine which attributes are driving patient preferences, the trade-offs between attributes that people are willing to accept and how changes in attributes can lead to changes in preferences and likely service uptake.

A simplified example from a UK survey of community preferences for alternative policy models of out of hours general practice contact $^{30}$ is shown in figure 1. Two unlabelled alternative healthcare delivery policies, option A and option B, were described using six different attributes, time to make initial contact, time waiting for advice or treatment, whether you are informed of the waiting time, face to face or telephone contact, whether you are seen by a doctor or nurse and likelihood contact relieves anxiety. The analysis will indicate individuals' preferences for out of hours care (option A and option B) based on the relative weight attached to attributes. For example, choosing option A would suggest that respondents were willing to wait an extra $55 \mathrm{~min}$ to be assessed and treated by a doctor rather than by a nurse.

By presenting respondents with a series of choices where the levels of the attributes are varied, researchers are able to quantify how these attributes influence choice. Given a sufficient number of choices to allow variation across all attributes, this approach enables estimates of the marginal effect of each attribute on choice and the marginal rate of substitution or trade-offs between attributes. In principle, this can be done by offering respondents choices using every combination of attributes, a 'full factorial' design. In practice, such a design is rarely feasible; efficient designs are therefore paramount, particularly when considering manifold choice options and interactions between attributes and socio-demographic characteristics on choice.

\section{STUDY METHODS}

Stage 1: establishing attributes using qualitative methods Qualitative one-on-one semi-structured interviews will be conducted with RACF residents, their families, staff of aged care facilities and ED staff. Maximal variation purposive sampling will be employed to ensure that a wide range of views are explored.

A semi-structured interview guide has been developed to investigate the key areas around which attributes for the DCE will be developed. We begin by asking contextual information on the respondents' previous experiences with an episode of care in the ED. Second, we seek evaluative information, asking the respondents to describe positive and negative perceptions concerning ED care and any alternative methods of acute care they have experienced. Specifically we explore the decisionmaking processes that went into seeking care, the technical quality of care delivery in terms of things such as waiting time and relief of symptoms and process factors such as comfort of the physical environment, attentiveness of staff and communication. Finally, we seek strategic data from respondents, asking them to describe their ideal care delivery policy and their attitude towards alternative policies. The total number of interviews conducted will be based upon reaching 'saturation point', when no new views are expressed, and subsequent participants repeat views expressed by previous participants. 'Saturation point' is often reached by the 20th interview. Respondent groups are likely to have different experiences; therefore, we will recruit up to 20
Figure 1 Example of a discrete choice question.

\begin{tabular}{|lll|}
\hline & Option $\mathrm{A}$ & Option B \\
\hline Time to make initial contact & $5 \mathrm{~min}$ & $5 \mathrm{~min}$ \\
\hline Time waiting for advice or treatment & $1 \mathrm{~h}$ & $5 \mathrm{~min}$ \\
\hline Informed of expected waiting time & Yes & Yes \\
\hline Type of contact is & In person & In person \\
\hline You are attended to by & Doctor & Specially trained nurse \\
\hline Chance contact relieves anxiety is & $50 \%$ & $50 \%$ \\
\hline & $\square$ & $\square$ \\
\hline
\end{tabular}


staff from ED, 20 staff from RACF (recognising RACF and ED staff may have similar views and saturation occur earlier), 20 RACF residents and 20 family members.

\section{Qualitative analysis}

All interviews will be recorded and transcribed and then analysed. Transcripts will be entered into NVIVO V.9 (QSR International Pty Ltd) and reviewed line by line by the study team. A preliminary coding system will be developed, using a phenomenological approach for thematic analysis as detailed by Crotty. ${ }^{31}$ Because of limited previous research in this area, findings from the content and thematic analyses will be essential to inform the design of the DCE and will form a fundamental and separate research output.

\section{Stage 2: design of discrete choice questionnaires}

Data from stage 1 , systematic reviews of the quantitative ${ }^{6}$ and qualitative literature on non-ED alternative models of care, and other research ${ }^{1632} 33$ will be used to inform DCE attributes. Once the attributes have been decided, a statistically efficient design for the discrete choice study will be created. This approach links statistical efficiency to the econometric model that is likely to be estimated from choice data using the design. ${ }^{34} 35$ This approach often lets go of the orthogonality constraint and attempts to minimise the expected asymptotic variance-covariance (AVC) matrix of the design. Efficient choice designs therefore attempt to maximise the likely asymptotic $t$-ratios obtained from choice data collected. As such, they attempt to minimise the correlation in the data for estimation purposes, and collect data such that parameter estimates have as small as possible standard errors. These designs make use of the fact that the AVC matrix (the roots of the diagonal of this matrix are the asymptotic standard errors) of the parameters can be derived if the parameters are known. Since the objective of the DCE is to estimate these parameters, they are unknown at the time of design. However, if some prior information about these parameters is available (eg, parameter estimates available in the literature from similar studies or parameter estimates from pilot studies), then this AVC matrix can be determined, assuming that the priors are correct.

The initial design will be tested with a small pilot study to assess survey comprehension. Results will be used to revise the wording and design of the study. This step is important because of the age of RACF respondents, though high acceptance levels and good reliability and validity of DCE in older people are anticipated. ${ }^{36}$

Once attribute descriptions are finalised, a second pilot study will be conducted to collect response data to calculate prior parameter estimates with which to inform the design of the main study. It is expected that approximately 20 respondents per group will be sufficient to calculate these priors. Based on these prior parameter estimates, the study design will be revised to maximise efficiency and validity.

\section{Stage 3: DCE survey}

Three respondent groups will be included: older people living in RACF, family members of aged care residents and staff of RACF. RACF that have taken part in stage 1 will also recruit respondents for the DCE. Quota sampling will be used to recruit a respondent sample broadly representative of the Australian RACF resident population (based upon characteristics such as age, sex, recent experience of an ED facility); family members of residents and staff of RACF will also be approached via RACF. Many residents in high-level RACF will have cognitive impairment, excluding them from participation in the DCE. This, however, reflects the reality of current decision making; care decisions for these residents are made on their behalf by staff, relatives or health professionals.

Respondents will either complete the DCE as a webbased survey or in a face-to-face setting using a computer-assisted personal interview (CAPI). Previous experience suggests that web-based surveys can be successfully utilised, even in older respondents, and provide a highly efficient means to collect data; we anticipate that most family member and RACF staff respondents will prefer to complete the DCE in this way. For those respondents preferring a face-to-face approach, particularly RACF residents, CAPIs can also be successfully implemented in a relatively efficient manner while ensuring data quality and timeliness. Upon consent, respondents will be asked to choose between the status quo option of ED and alternative unlabelled acute care delivery options, which vary across a range of attributes. Final respondent numbers required for the DCE will depend on the number of attributes and the range of each attribute and the prior parameter estimates identified from stages 1 and 2 .

\section{Sample size}

The current theory of sampling for these experiments does not directly address the issue of minimum sample size requirements in terms of the reliability of the parameter estimates produced in the design of stated choice experiments (see eg, Hensher et $a l^{37}$ and Louviere $e t a p^{38}$ ). Rather, sampling theory as applied to choice modelling is designed to minimise the error in the choice proportions of the alternatives under study. ${ }^{3435}$ This means that the final sample size required is based upon the characteristics of the design itself such as the number of attributes included, the attribute level range, the number of choice scenarios presented, the number of alternatives in each choice set and the size and direction of prior parameters obtained from the pilot study.

We expect a sample size of approximately 200 respondents from each respondent group. With an efficient choice design, this sample size will likely be sufficient to assess any differences in preference structure between respondent groups and across age, sex and other demographic characteristics. 
Stage 4: analysis

A mixed multinomial logit (MMNL) (also known as random parameters logit) model using a panel size specification will be used for each analysis. A panel specification of the model allows for non-independence of observations provided by the same respondent, that is, it can account for correlations among the multiple choices made by the same individual. MMNL models relax certain statistical assumptions of more commonly used multinomial logit (MNL) models and often lead to models that better explain choice behaviour. ${ }^{37} 39$ In MNL choice models, commonly used in health economics, parameters associated with each attribute are treated as fixed. These fixed values are the average (or point estimates) associated with a population-level distribution; other information in the distribution is not considered. An MMNL allows consideration of the full distribution of a parameter estimate, and the fixed parameter becomes a random parameter. 'Random parameter' simply implies that each individual has an associated parameter estimate on that specified distribution. While the exact location of each individual's preferences on the distribution may not be known, estimates of 'individual-specific preferences' can be accommodated by deriving the individual's conditional distribution, based-within sample-on their choices (ie, prior knowledge).$^{39}$ Interactions between attributes in the discrete choice surveys and between attributes and population characteristics (eg, age, gender, income, education, prior ED experience) will be explored in the mixed logit analysis.

Model results will expressed as parameter estimates $(\beta)$, the odds of choosing one option instead of another (and 95\% CIs of the ORs) and $\mathrm{p}$ values. Acceptable trade-offs between attributes will also be calculated.

\section{ETHICAL CONSIDERATIONS}

The PROSpER Study has been approved by the University of Sydney, Human Research Ethics Committee (Protocol numbers 10653 and 14382) and the Royal Perth Hospital Ethics Committee (reference 2009/045).

Confidentiality and anonymity of the data will be strictly maintained. Respondent interviews, including face-to-face CAPI for the DCE, will only take place after written informed consent is obtained from participants. Participants will not be identifiable in any transcripts or in any publications. It will be made clear to all participants that they have the right to withdraw from the research at any point in time.

For respondents choosing to complete the survey online, written consent is not possible. As such participant information for the online survey will include the following statement "Being in this study is completely voluntary-you are not under any obligation to consent and-if you do consent-you can withdraw at any time without affecting your relationship with The University of Sydney. By completing the survey you have consented to be part of the study. You may stop completing the online survey at any point if you do not wish to continue, and we will not use your answers. Once you have submitted your survey anonymously, your responses cannot be withdrawn."

\section{DISSEMINATION}

The results will be published in internal reports, peerreviewed scientific journals and via conference presentations; a newsletter will also be provided to study participants who are interested. At the conclusion of the study, we will also hold a stakeholder roundtable to discuss the results and subsequent implementation strategies.

\section{DISCUSSION}

The results from this survey will inform health policy by highlighting the factors that influence preferences for how acute care services should be designed for older Australians. Our analysis will directly address the aims of this research project by providing:

- Estimates of the marginal effect (importance) of each attribute on overall preference for each policy.

- Estimates of marginal rates of substitution between attributes based on the ratio of parameter estimates, giving an indication of the extent to which respondents are prepared to trade-off one attribute for another, for example, if waiting time and ability to be treated by a specialist emergency physician are attributes, the marginal rates of substitution between these reflects the waiting time people are willing to accept in order to be seen by a specialist.

- An indication of the predicted values or 'market shares' associated with different parameter levels within the estimated utility functions. This allows forecasting of the likely uptake of various policy options, given particular policy characteristics.

- An understanding of the relationship between resident, family and provider preferences in acute care decisions.

These outputs will inform the development of health policy regarding the provision of acute care services for older people living in RACF. Given that separate surveys will be carried out across multiple stakeholder groups, an analysis of whether the preferences of particular groups are under- or over-represented in current practice will be assessed. By understanding the trade-offs and likely uptake of alternative models of care provision, we will be able to guide delivery of health services such that they are responsive to consumer preferences.

Contributors GA and $\mathrm{KH}$ were responsible for the conceptual design of the study. All authors participated in revisions to the study design and approved the final study design. All authors were involved in drafting and revising the manuscript. All authors are involved in the implementation of the project and have read and approved the final manuscript.

Funding The PROSpER Study is funded by the Australian Research Council Discovery Project grant number DP120100770. The funders have no role in study design; collection, management, analysis and interpretation of data; nor in writing of any reports or the decision to submit the reports for publication.

Competing interests None. 
Ethics approval Ethics approval was provided by University of Sydney Human Research Ethics Committee; Royal Perth Hospital Ethics Committee.

Provenance and peer review Not commissioned; internally peer reviewed.

Data sharing statement No additional data available.

\section{REFERENCES}

1. AlHW. Residential Aged Care in Australia 2007-08: A Statistical Overview. Canberra: Australian Institute of Health and Welfare, 2009. Report No: Number 28.

2. McCloskey R, van den Hoonaard D. Nursing home residents in emergency departments: a Foucauldian analysis. J Adv Nurs 2007:59:186-94.

3. Ouslander JG. Medical care in the nursing home. JAMA 1989;262:2582-90.

4. Mosby's Medical Dictionary. 8th edn. Chatswood, Australia: Elsevier Publishing, 2008.

5. National Health and Hospitals Reform Commission. A Healthier Future for All Australians-Final Report of the National Health and Hospitals Reform Commission-June 2009. Canberra, ACT: Commonwealth of Australia, 2009.

6. Arendts G, Howard K. The interface between residential aged care and the emergency department: a systematic review. Age Ageing 2010;39:306-12.

7. Kerr HD, Byrd JC. Nursing home patients transferred by ambulance to a VA emergency department. J Am Geriatr Soc 1991;39:132-6.

8. Chin MH, Jin L, Karrison TG, et al. Older patients' health-related quality of life around an episode of emergency illness. Ann Emerg Med 1999;34:595-603.

9. Denman SJ, Ettinger WH, Zarkin BA, et al. Short-term outcomes of elderly patients discharged from an emergency department. J Am Geriatr Soc 1989;37:937-43.

10. Wilber ST, Gerson LW, Terrell KM, et al. Geriatric emergency medicine and the 2006 Institute of Medicine reports from the Committee on the Future of Emergency Care in the U.S. health system. Acad Emerg Med 2006;13:1345-51.

11. Hwang U, Morrison RS. The geriatric emergency department. J Am Geriatr Soc 2007;55:1873-6.

12. Nerney MP, Chin MH, Jin L, et al. Factors associated with older patients' satisfaction with care in an inner-city emergency department. Ann Emerg Med 2001;38:140-5.

13. Creditor MC. Hazards of hospitalization of the elderly. Ann Intern Med 1993;118:219-23.

14. Inouye SK, Charpentier PA. Precipitating factors for delirium in hospitalized elderly persons. Predictive model and interrelationship with baseline vulnerability. JAMA 1996;275:852-7.

15. Gardam MA, Amihod B, Orenstein P, et al. Overutilization of indwelling urinary catheters and the development of nosocomial urinary tract infections. Clin Perform Qual Health Care 1998;6:99-102.

16. Arendts $\mathrm{G}$, Reibel $\mathrm{T}$, Codde $\mathrm{J}$, et al. Can transfers from residential aged care facilities to the emergency department be avoided through improved primary care services? Data from qualitative interviews. Australas J Ageing 2010;29:61-5.

17. Campbell MK, Silver RW, Hoch JS, et al. Re-utilization outcomes and costs of minor acute illness treated at family physician offices, walk-in clinics, and emergency departments. Can Fam Physician 2005:51:82-3.

18. Kurrle SE. Improving acute care services for older people. Med J Aust 2006;184:427-8.

19. Kane RL, Keckhafer G, Flood S, et al. The effect of Evercare on hospital use. J Am Geriatr Soc 2003;51:1427-34.
20. Codde J, Arendts G, Frankel J, et al. Transfers from residential aged care facilities to the emergency department are reduced through improved primary care services-an intervention study. Australas $J$ Ageing 2010;29:150-4

21. Bellantonio $\mathrm{S}$, Kenny $\mathrm{AM}$, Fortinsky $\mathrm{RH}$, et al. Efficacy of a geriatrics team intervention for residents in dementia-specific assisted living facilities: effect on unanticipated transitions. J Am Geriatr Soc 2008;56:523-8

22. Hui E, Woo J, Hjelm M, et al. Telemedicine: a pilot study in nursing home residents. Gerontology 2001;47:82-7.

23. Montalto M. Hospital in the nursing home: treating acute hospital problems in nursing home residents using a Hospital in the Home model. Aust Fam Physician 2001;30:1010-12.

24. Salvi F, Morichi V, Grilli A, et al. A geriatric emergency service for acutely ill elderly patients: pattern of use and comparison with a conventional emergency department in Italy. J Am Geriatr Soc 2008;56:2131-8.

25. Bridges JF, Hauber AB, Marshall DA, et al. Conjoint analysis applications in health-a checklist: a report of the ISPOR Good Research Practices for Conjoint Analysis Task Force. Value Health 2011;14:403-13.

26. Lancsar E, Louviere J. Conducting discrete choice experiments to inform healthcare decision making: a user's guide. Pharmacoeconomics 2008;26:661-77.

27. Bridges JF, Kinter E, Kidane L, et al. Things are looking up since we started listening to patients: recent trends in the application of conjoint analysis in health 1970-2007. Patient 2008; $1: 273-82$

28. Marshall DA, Bridges JF, Hauber AB, et al. Conjoint analysis applications in health-how are studies being designed and reported? An update on current practice in the published literature between 2005 and 2008. Patient 2010;3:249-56.

29. Arendts G, Howard K, Rose JM. Allocation decisions and patient preferences in emergency medicine. Emerg Med $\mathrm{J}$ 2011;28:1051-4.

30. Gerard K, Lattimer V, Surridge $\mathrm{H}$, et al. The introduction of integrated out-of-hours arrangements in England: a discrete choice experiment of public preferences for alternative models of care. Health Expect 2006;9:60-9.

31. Crotty M. Phenomenology and Nursing Research. South Melbourne: Churchill Livingstone, 1996.

32. Arendts G, Dickson C, Howard K, et al. Transfer from residential aged care to emergency departments: an analysis of patient outcomes. Intern Med J 2012;42:75-82.

33. Codde J, Frankel J, Arendts G, et al. Quantification of the proportion of transfers from residential aged care facilities to the emergency department that could be avoided through improved primary care services. Australas J Ageing 2010;29:167-71.

34. Rose JM, Bliemer MCJ. Constructing efficient stated choice experimental designs. Transport Rev 2009;29:587-617.

35. Bliemer MCJ, Rose JM. Construction of experimental designs for mixed logit models allowing for correlation across choice observations. Transportation Res B 2010;46:720-34.

36. Ratcliffe J, Laver K, Couzner L, et al. Not just about costs: the role of health economics in facilitating decision making in aged care. Age Ageing 2010;39:426-9.

37. Hensher DA, Rose JM, Greene WH. Applied Choice Analysis: A Primer. 1st edn. Cambridge: Cambridge University Press, 2005.

38. Louviere J, Hensher DA, Swait JD. Stated Choice Methods-Analysis and Application. Cambridge: Cambridge University Press, 2000.

39. Hensher DA, Greene WH. Mixed logit models: state of practice. Transportation 2003;30:133-76. 